\title{
N6-methyladenosine-dependent modification of circGARS acts as a new player that promotes SLE progression through the NF- $\mathrm{kB} / \mathrm{A} 20$ axis
}

Xingwang Zhao ${ }^{1 \dagger}$, Rui Dong ${ }^{2 \dagger}$, Longlong Zhang ${ }^{3}$, Junkai Guo ${ }^{1}$, Ying Shi ${ }^{4}$, Lan Ge', Juan Wang ${ }^{1}$, Zhiqiang Song ${ }^{1}$, Bing $\mathrm{Ni}^{5 *}$ and $\mathrm{Yi} \mathrm{YOu}^{1 *}$ (D)

\begin{abstract}
Background: Certain circRNAs could be used as biomarkers to determine the risk of development and/or severity of systemic lupus erythematosus, and their new function in the regulation of gene expression has motivated us to investigate their role in SLE
\end{abstract}

Methods: Experimental methods including qRT-PCR, RNA immunoprecipitation (RIP), pulldown, dual luciferase reporter assay, RNA interference and cell transfection, RNA fluorescence in situ hybridization, western blotting, and mass spectrometry were used to assessed circGARS (hsa_circRNA_0009000) for immune functions and defined mechanisms by which circGARS promotes the progression in SLE.

Results: Our results demonstrated that the levels of circGARS was remarkably upregulated in SLE and correlated with clinicopathological features. CircGARS directly combined with microRNA-19a (miR-19a). Functionally, circGARS downregulated the expression of TNFAIP3 (A20, tumor necrosis factor alpha-induced protein 3) to mediate the activation of immune responses that were regulated by the nuclear factor-kB (NF-kB) pathway as a negative feedback mechanism. In addition, miR-19a regulated A20 (TNFAIP3) degradation by downregulating the expression of YTH N6-methyladenosine RNA-binding protein 2 (YTHDF2).

Conclusions: The circGARS sponges miR-19a to regulate YTHDF2 expression to promote SLE progression through the A20/NF-KB axis and may act as an independent biomarker to help the treatment of SLE patients.

Keywords: circGARS, miR-19a, A20, m6A, YTHDF2, Systemic lupus erythematosus

*Correspondence: nibing@tmmu.edu.cn; youyi_cq@163.com

tXingwang Zhao and Rui Dong contributed equally to this work.

${ }^{1}$ Department of Dermatology, Southwest Hospital, Army Medical

University (Third Military Medical University), Chongqing, China

${ }^{5}$ Department of Pathophysiology, College of High Altitude Military

Medicine, Army Medical University (Third Military Medical University),

Chongqing, China

Full list of author information is available at the end of the article

\section{Background}

Systemic lupus erythematosus (SLE) is a chronic and systemic autoimmune disease with diverse clinical manifestations and complicated disease progression. The pathogenesis of SLE are still largely unknown. A highly complicated interaction among various environmental factors and genetic susceptibility is most likely involved $[1,2]$. Although mortality for patients with SLE has greatly declined, accurate diagnosis and better management of complications, especially the development of original author(s) and the source, provide a link to the Creative Commons licence, and indicate if changes were made. The images or other third party material in this article are included in the article's Creative Commons licence, unless indicated otherwise in a credit line to the material. If material is not included in the article's Creative Commons licence and your intended use is not permitted by statutory regulation or exceeds the permitted use, you will need to obtain permission directly from the copyright holder. To view a copy of this licence, visit http://creativecommons.org/licenses/by/4.0/. The Creative Commons Public Domain Dedication waiver (http://creativeco mmons.org/publicdomain/zero/1.0/) applies to the data made available in this article, unless otherwise stated in a credit line to the data. 
safer and more effective therapies, are still urgent. Therefore, there is an urgent need to gain an in-depth understanding the pathogenesis of SLE.

MicroRNAs (miRNAs) and long noncoding RNAs (lncRNAs) are two main kinds of noncoding RNAs that regulate the initiation and development of SLE [3, 4]. CircRNA (circRNA) is a novel non-coding RNA with a unique covalent closed-loop structure, which makes it an excellent diagnostic marker for SLE $[5,6]$. In recent years, more functional circRNAs have been discovered using high-throughput sequencing analysis and bioinformatic methods, and circRNAs have been shown to play an important role in the progression of autoimmune diseases. Moreover, circRNAs have been revealed to act as microRNA (miRNA) sponges, participating in binding to RNA-binding proteins and protein translation [7-9]. Studies have reported that numerous circRNAs are associated with SLE, such as hsa_circ_0000479, hsa circ_0068367, and hsa_circ_0044235 [10, 11]. However, the exact molecular mechanism remains to be elucidated.

m6A RNA modification is a widespread reversible dynamic modification in eukaryotic cells and is one of the most abundant mRNA nonterminal modifications. YTHDF2 protein can directly recognize m6A; the levels of YTHDF2 mRNA are decreased in peripheral blood from patients with SLE, which might be risk factors for SLE $[12,13]$. TNF- $\alpha$-induced protein 3 (TNFAIP3, also known as A20) is significantly downregulated in peripheral blood mononuclear cells (PBMCs) of SLE patients. It is one of the major SLE susceptibility genes involved in the negative regulation of inflammatory responses through modulation of the NF-kB pathway [14]. Genetic polymorphisms analysis suggests that the miR-17-92 cluster closely related with SLE of Chinese population [15]. The miR-17-92 cluster member miR-19a play an important role in multiple autoimmune patients. miR-19a inhibited expression of several targets including SOCS1 and A20 [16-18]. The regulatory effects of miR-19a on TNFAIP3 (A20) and NF- $k B$ signaling have been reported, which involved in immune inflammatory responses of SLE [19-21]. Considering the role of m6A modification in regulating gene expression and immune response, the association between $\mathrm{m} 6 \mathrm{~A}$ modification and SLE remains to be clarified. It is possible that m6A modification may be involved in SLE etiology and participate in the initiation and progression of SLE [22]. However, there are relatively few studies on circRNAs in this area.

Herein, our aim was to analyze the molecular mechanisms of circRNAs in SLE patients. circGARS was specifically hyperexpression and closely related to the disease activities of SLE patients. To investigate whether the abnormal activation of the A20/NF- $\mathrm{kB}$ signaling pathway was regulated by circGARS, we studied how
circGARS enhanced transcriptional levels by directly binding to m6A modification proteins, which might participate in the pathogenesis of SLE. We concentrated on the effect of circGARS competitively binding to miR-19a and regulating the expression of YTHDF2, which may regulate A20 degradation and eventually promote disease progression. Our study may provide a potential biomarker for SLE patients and/or a promising therapeutic target against SLE.

\section{Materials and methods Clinical samples}

We collected 62 samples of SLE patients and healthy volunteers from the First Affiliated Hospital of Army Medical University from September 2017 to January 2020. Patients who met four or more American College of Rheumatology (ACR) SLE criteria, as revised in 1997, were included. Demographic, clinical and laboratory characteristics were recorded for each subject and disease activity was assessed using the Systemic Lupus Erythematosus Disease Activity Index (SLEDAI) at blood drawing. The information about the SLE patients can be found in Additional File 1: Table S1 and Additional File 2: Table S2.

\section{RNA extraction and RT-qPCR}

The whole blood $(10 \mathrm{ml})$ was collected in the anticoagulant tube from each subject, and PBMCs (peripheral blood mononuclear cells) were isolated by density-gradient centrifugation using Ficoll-Paque Plus (GE Healthcare Biosciences) within $4 \mathrm{~h}$ of collecting the samples. Total RNA was harvested and separated from PBMCs of samples via TRIzol reagent (Invitrogen). Extracted total RNA was reverse-transcribed to complementary DNA (cDNA) using Prime Script RT Master Mix (Takara, Japan) with random or oligo (dT) primers. SYBR Green SuperMix (Roche, Basel, Switzerland) was used for qRTPCR. The relative expression levels were detected by the $2^{-\Delta \Delta \mathrm{Ct}}$ method. The primers are listed in Additional File 3: Table S3.

\section{Ribonuclease $\mathrm{R}$ treatment}

Two micrograms of total RNA from PBMCs was mixed with or without $3 \mathrm{U} / \mu \mathrm{g}$ ribonuclease $\mathrm{R}$ (Epicentre Technologies, Madison, WI, USA) at $37^{\circ} \mathrm{C}$ for $20 \mathrm{~min}$. Then, the samples were purified with an RNeasy MinElute Cleaning Kit (74204, Qiagen, Germany) and analyzed by RT-PCR. The stability of circGARS and linear GARS was determined.

\section{Fluorescence in situ hybridization (FISH)}

RNA in situ hybridization was performed using specific probes for circGARS and miR-19a-5p. The circGARS 
probe for FISH was 5'-ATCCTTCTTATATGCCTT AC-3'. The experiment followed the manufacturer's instructions for Alexa Fluor ${ }^{\mathrm{TM}} 488$ Tyramide SuperBoost $^{\mathrm{TM}}$ Kits by Riobio (Guangzhou, Guangdong, China). Confocal microscopy was used to better visualize the presence of circGARS and miRNA-19a-5p.

\section{PBMCs isolation and culture}

$1 \times 10^{6}$ PBMC cells were cultured in RPMI Media 1640 (Invitrogen-Gibco, USA) supplemented with 10\% fetal bovine serum (Invitrogen-Gibco, USA), 100 U/mL penicillin, $100 \mathrm{U} / \mathrm{mL}$ streptomycin (Invitrogen-Gibco, USA), and $1.5 \mathrm{mg} / \mathrm{L}$ glutamine and incubated at $37^{\circ} \mathrm{C}$ with $5 \%$ $\mathrm{CO}_{2}$ for $24 \mathrm{~h}$ before transfection.

\section{Plasmid construction and cell transfection}

Cells were separately transfected with Lipofectamine 3000 (Invitrogen, Carlsbad, CA). The circGARS overexpression plasmids and empty vector were constructed by GeneChem (Shanghai, China). The miR-19a-3p/5p mimics/inhibitor, miRNA-NC mimics/inhibitor, siRNA for circGARS, and siRNA-NC were chemically synthesized by Riobio (Guangzhou, Guangdong, China). The sequence of siRNA for circGARS was 5-ATGAGAAAGGGGTTGGATTGA-3. The transfection process lasted $48 \mathrm{~h}$.

\section{Luciferase reporter gene assay}

For the circGARS and miR-19a-5p luciferase reporter gene assays, the circGARS sequences containing wildtype miR-19a-5p predicted binding sites were inserted into the region directly downstream of a cytomegalovirus (CMV) promoter-driven firefly luciferase cassette in a pCDNA3.1 vector by GeneChem (Shanghai, China). For the YTHDF2 $3^{\prime}$-UTR and miR-19a-3p luciferase reporter gene assay, the YTHDF2 3 '-UTR sequences containing wild-type miR-19a-3p predicted binding sites were inserted into the region directly downstream of a T7 promoter-driven firefly luciferase cassette in a psiCHECK $^{\mathrm{TM}}$-2 vector (Promega, Madison, USA). All constructs were detected by sequencing. $2 \times 10^{5} 293 \mathrm{~T}$ cells were seeded into 24-well plates and co-transfected with a mixture of $1 \mu \mathrm{g}$ of luciferase reporter and miRNA mimics and inhibitor. After $48 \mathrm{~h}$ of incubation, the firefly and Renilla luciferase activities were quantified using the Dual Luciferase Assay System (Promega, Madison, WI, USA).

\section{Quantification of the m6A modification}

Concentrations of $\mathrm{m} 6 \mathrm{~A}$ in PBMC supernatants were analyzed by an Epiquik m6A RNA Methylation Quantification Kit (colorimetric) following the manufacturer's instructions. The absorbance was measured at $450 \mathrm{~nm}$ using a microplate analyzer and the horizontal colorimetric value of $\mathrm{m} 6 \mathrm{~A}$ was measured according to the standard curve.

\section{Prediction of ceRNAs for circGARS}

To explore the functions of the candidate disease stagerelated circGARS, the potential miRNA binding sites were predicted. We used circMir1.0 software to identify circGARS-targeting miRNAs, which indicates a higher probability of being a putative ceRNA for circGARS.

\section{RNA-binding protein immunoprecipitation (RIP)}

A Magna RIP RNA-Binding Protein Immunoprecipitation Kit (Millipore, Billerica, MA) was used to perform the RIP experiments according to the manufacturer's instructions. Human AGO2 antibody (ab57113, Abcam, Cambridge, MA) was used for RIP. In brief, the PBMCs was harvested and dissolved on ice in the RIP lysis buffer for $30 \mathrm{~min}$. After centrifugal collection, the supernatant was incubated with $30 \mu$ l Protein-G agarose beads (Roche, USA) and 8ul AGO2 antibodies. After overnight incubation, the complexes were centrifuged and then washed six times using washing buffer. The bead-bound proteins were analyzed by western blotting. Co-precipitated RNA was detected by qRT-PCR.

\section{RNA pulldown}

The biotin-coupled RNA complex was pulled down by incubating the cell lysates with Pierce $^{\mathrm{TM}}$ Streptavidin Magnetic Beads (Thermo Fisher Scientific, USA) as per the manufacturer's protocol. The enrichment of circGARS in the capture fractions was detected by qRTPCR analysis. The circGARS junction probe was $5^{\prime}$-CAG CACATCCAACAATCTCA- $3^{\prime}$, and the control probe was 5'-TTGTACTACACAAAAGTACTG-3' (ordered from Sangon Biotech, Shanghai, China). miRNAs were detected by RT-PCR. The probe sequences used are listed in Additional File 4: Table S4.

\section{Western blot analysis}

RIPA lysis buffer (Beyotime, Shanghai, China) supplemented with protease inhibitors (P8340, Sigma-Aldrich) was used to lyse the total protein from PBMCs. The protein concentration was determined using a Braford Protein Assay Kit (Beyotime, Shanghai, China). Total cell lysates were separated by $10 \%$ sodium dodecyl sulfate polyacrylamide gel electrophoresis (SDS-PAGE) and then transferred to polyvinylidene fluoride membranes (Millipore, Bradford, MA, USA). Membranes were blocked with milk for $2 \mathrm{~h}$ on a shaker at room temperature followed by incubation with primary antibodies overnight at $4{ }^{\circ} \mathrm{C}$. The primary antibodies used were mouse antiArgonaute-2 (ab57113, Abcam, 1:1000), rabbit anti-A20 
(5630S, Cell Signaling Technology, CST, 1:1000), rabbit anti-NF-kB (8242 T, CST, 1:1000), rabbit anti-p-NF- $\mathrm{kB}$ (3033 T, CST, 1:1000), rabbit anti-IkB $\alpha$ (9242S, CST, 1:1000), rabbit anti-p-IkB $\alpha$ (2859S, CST, 1:1000), rabbit anti-YTHDF2 (80014S, CST, 1:1000), rabbit anti- $\beta$-actin (4970S, CST, 1:1000), and GAPDH antibody (5174S, CST, 1:1000), which were used as controls. After washing with PBST three times, the blots were then incubated with horseradish peroxidase-coupled anti-rabbit antibody (7074S, CST, 1:1000) or anti-mouse antibody (7076S, CST, 1:1000) for $1 \mathrm{~h}$ at room temperature. The blot signal was examined by Pierce ECL western blotting Substrate (Thermo Fisher Scientific, USA) with a ChemiDoc ${ }^{\mathrm{TM}}$ Touch Imaging System (Bio-Rad, USA) according to the manufacturer's recommendations. The integrated density values were calculated using Quantity One software (BioRad, USA).

\section{Statistical analysis}

GraphPad Prism version 8.0 (GraphPad Software, La Jolla, CA) was used to prepare graphs and presented as the mean \pm standard deviation. The difference was determined via the paired Student's $t$ test. All experiments mentioned above were repeated three times independently. A two-tailed $P$ value $<0.05$ was considered statistically significant.

\section{Results}

Identification and characterization of circGARS, a circRNA specifically and highly expressed in SLE

To identify circRNAs specifically functioning in SLE, we analyzed the circRNA RNA-seq data to identify circRNAs with significant differences $(P<0.01)$ between healthy controls and SLE patients [23]. We selected and identified 10 upregulated circRNAs and 10 downregulated circRNAs (|fold change $\mid>2, P<0.01$, Additional File 5: Fig. S1 and Additional File 6: Table S5) significantly related to SLE. The results showed that circGARS (known as hsa_circ_0009000) had a higher level of expression in SLE patients. We further analyzed 62 patient samples and 62 healthy samples. As shown in Fig. 1a, circGARS showed significant higher expression levels in SLE patient samples than in healthy samples. CircGARS is a 254-nt circRNA, derived from the backsplicing of preRNA of the GARS gene, involving exons 11-12, as shown in Fig. 1b. The back-spliced junction point of circGARS was verified by sanger sequencing (Fig. 1c). Stability was measured using exonuclease RNase R, circGARS was highly resistant to RNase $\mathrm{R}$ digestion, whereas the linear RNA of GARS and $\beta$-actin was highly resolved (Fig. 1d). FISH results showed that circGARS was mainly localized in the cytoplasm (Fig. 1e). To evaluate the potential of circGARS as a biomarker for SLE, data on clinicopathological parameters were collected in 62 patients with SLE, and the correlation between these parameters and circGARS expression level was tested. Patients with high levels of circGARS had a significantly higher SLEDAI (Fig. 1f) and lower complement C3 levels (Fig. 1g). To evaluate the diagnostic value of circGARS for SLE, we performed receiver operating characteristic (ROC) curve analysis in 62 SLE patients and healthy controls to distinguish between SLE patients and HC. The area under the curve (AUC) was $0.8932(95 \% \mathrm{CI}=0.8369-0.9495)$ (Fig. 1h).

\section{CircGARS functions as a miR-19a sponge}

To discover the potential functional mechanism of circGARS, we used circMir1.0 software (http://www.bioinf. com.cn/) to analyze the potential target miRNAs that could bind with circGARS (Fig. 2a). Subsequently, we purified the circGARS-bound RNA complexes and confirmed the enrichment of miR-19a-5P. RNA pulldown assays suggested that miR-19a-5p may directly bind to circGARS (Fig. 2b). The regulatory effects of miR19a on TNFAIP3 (A20) and NF- $\mathrm{kB}$ signaling have been reported $[19,20]$. A20 is a ubiquitin-editing molecule, and it inhibits NF- $\mathrm{KB}$ activation and TNF-mediated apoptosis. This protein is involved in cytokine-mediated immune and inflammatory responses. Diseases associated with TNFAIP3 include autoinflammatory syndrome, especially SLE [21]. FISH analysis confirmed that miR$19 a-5 p$ was colocalized with circGARS in the cytoplasm (Fig. 2c). We next preformed argonaut 2 (AGO2) immunoprecipitation to determine whether circGARS served as a platform for AGO2 and miR-19a-5p. The results of AGO2 RNA-binding protein immunoprecipitation (RIP) assay (Fig. 2d) and qRT-PCR (Fig. 2e) supported this observation. To further test whether miR-19a-5p is a target of circGARS, wild-type or mutant circGARS was cloned into a luciferase vector (Fig. 2f) and then cotransfected with miR-19a-5p mimics and inhibitor into $293 \mathrm{~T}$ cells. The repeated dual luciferase experiments showed that miR-19a-5p significantly decreased the luciferase signal of the wild-type circGARS reporter (Fig. 2g, h). The results demonstrated that circGARS binds with miR-19a-5p.

\section{MiR-19a expression was downregulated and correlated} with clinicopathological characteristics in patients with SLE To investigate the role of miR-19a-5p in SLE, we used qRT-PCR to detect the expression of miR-19a-5p in PBMCs of 36 SLE patients and 25 healthy controls. The results demonstrated that the expression of miR-19a-5p was significantly downregulated in 


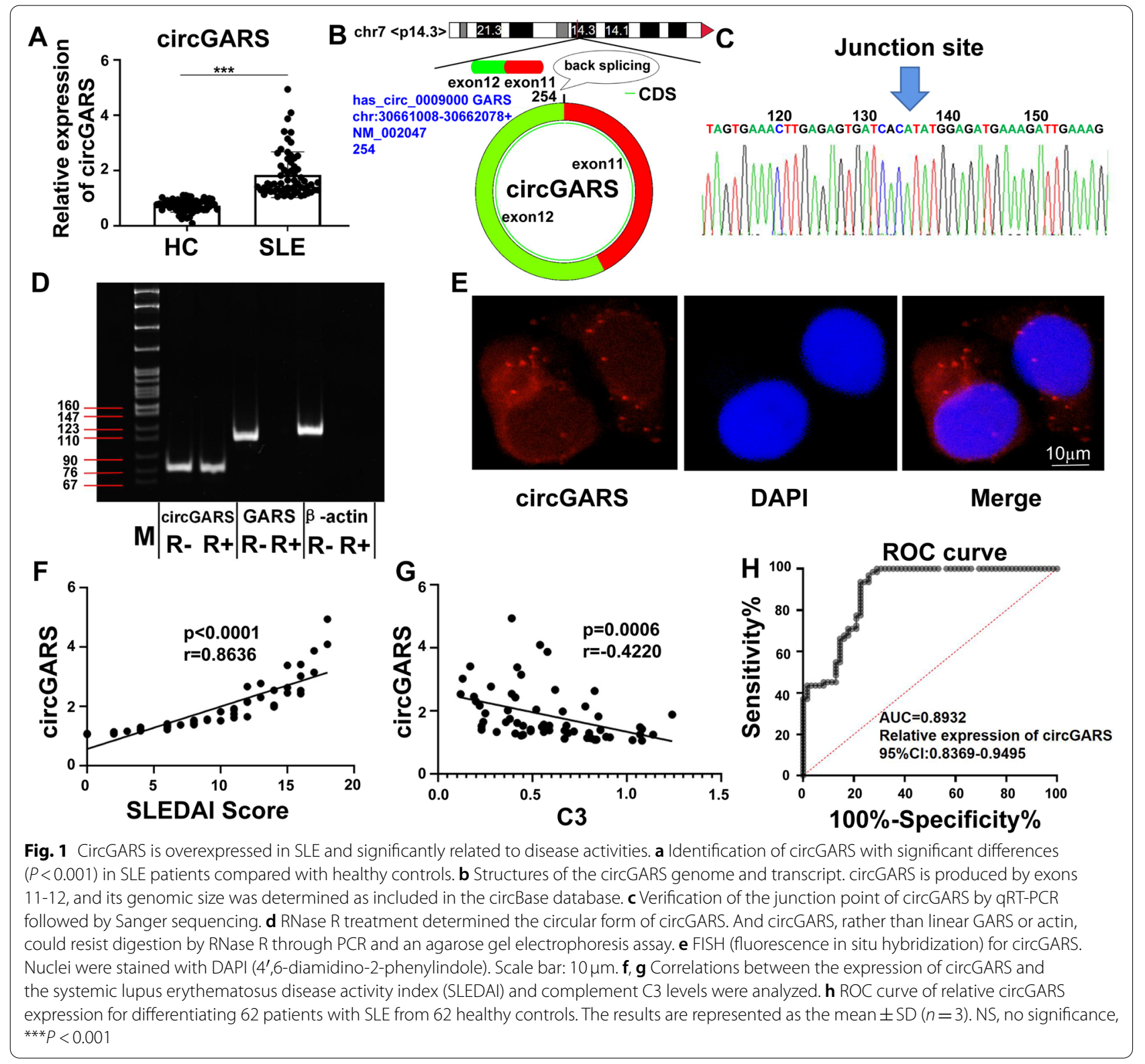

SLE (Fig. 3a). We examined the correlation between disease activity and miR-19a-5p expression, and the results showed that there was a strong inverse correlation between miR-19a-5p expression and the SLEDAI in SLE patients (Fig. 3b). miR-19a-5p expression was also positively correlated with $\mathrm{C} 3$ levels (Fig. 3c). In order to evaluate the diagnostic value of miR-19a-5p in SLE, ROC curves on relative expression of miR$19 a-5 p$ in 36 patients and 25 healthy controls were also analyzed. The AUC was 0.7589 (95\% CI = 0.63850.8793) (Fig. 3d). These results indicated that the expression of $\mathrm{miR}-19 \mathrm{a}-5 \mathrm{p}$ related to the disease activity of SLE, and miR-19a-5p could act as a biomarker to evaluate the activity of SLE and validate the effectiveness of SLE treatment.

\section{CircGARS sponged miR-19a to suppress the expression} of the $\mathrm{m} 6 \mathrm{~A}$ reader YTHDF2 to regulate $\mathrm{A} 20$ degradation Although we already know a lot about the m6A modification, the association between $\mathrm{m} 6 \mathrm{~A}$ modification and SLE remains to be clarified. To investigate whether m6A modification may be involved in SLE etiology and participated in the disease progress of SLE, we analyzed the degree of global m6A RNA methylation level in SLE patients, and the m6A RNA methylation level was significantly higher than that of the control 


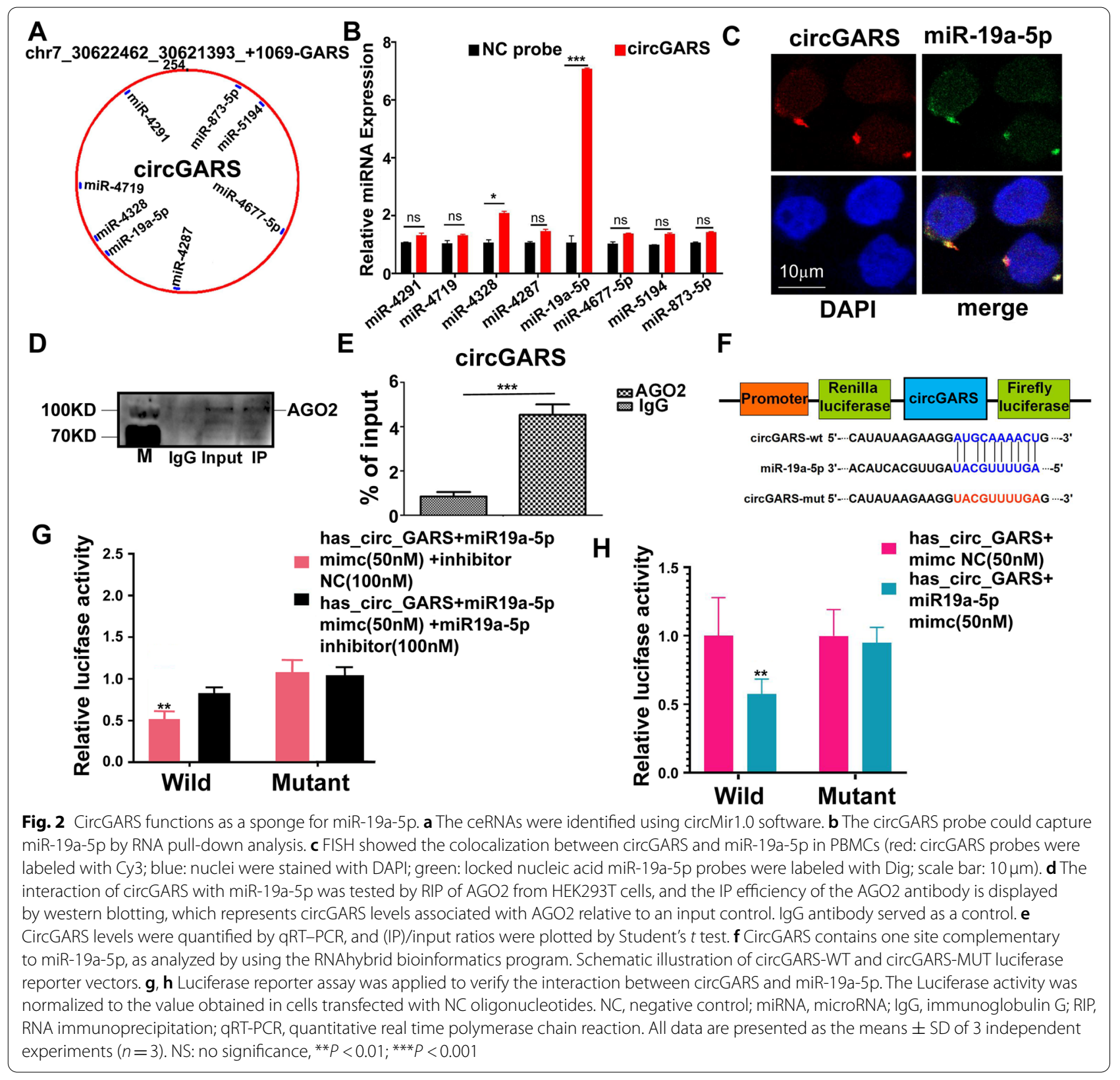

group (Fig. 4a). The abnormal expression of m6A modification proteins may directly affect the global m6A level and thus lead to the alteration of the expression of key immune-related genes and the occurrence of SLE. Therefore, examining the expression of proteins responsible for m6 A modification should provide insight on this possible mechanism. In addition, several miRNAs regulate the pathogenesis of SLE and show potential in the treatment of SLE. YTHDF2, an m6A reader protein, plays an important biological role in the regulation of mRNA m6A [24]. YTHDF2 selectively recognizes mRNA m6A to regulate mRNA stability, and the degradation of mRNAs can lead to the decrease of m6A levels [25]. We performed bioinformatics analysis to predict the potential miRNAs targeting the $3^{\prime}$-UTR of YTHDF2 by using the website TargetScan (www.targetscan.org/). MiR-19a was one of the candidates that was predicted (Fig. 4b) and conserved on the website. The Western blot analysis showed that miR-19a-3p could significantly inhibited the expression of YTHDF2 in PBMCs (Fig. 4c), suggesting that miR-19a-3p is involved in the regulation of YTHDF2 mRNA. Therefore, we concluded that YTHDF2 may be one of the target genes of 
A

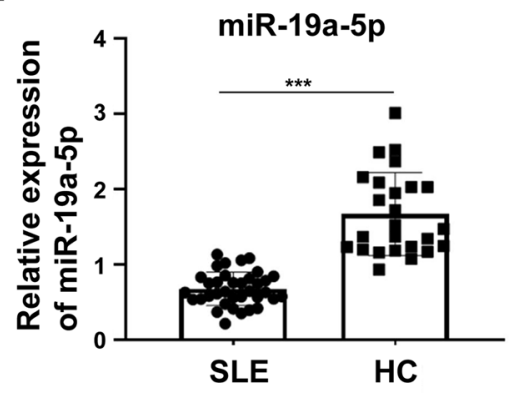

C

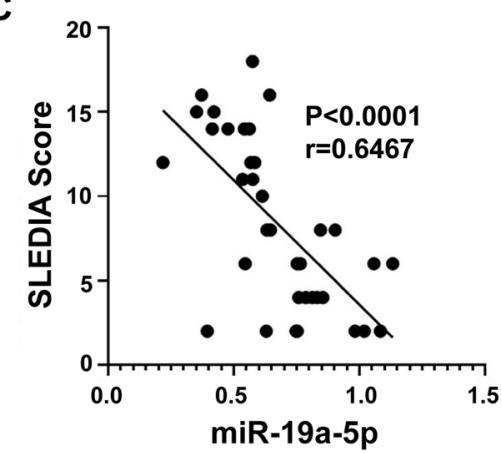

B

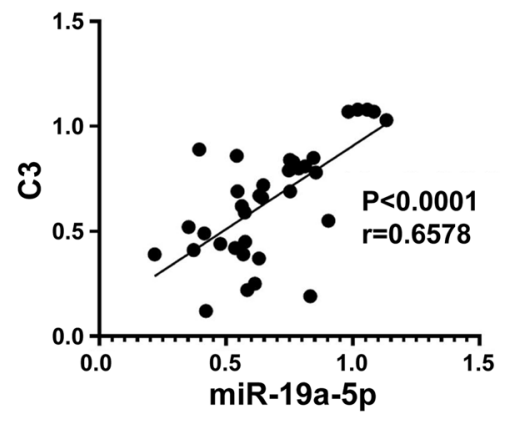

D

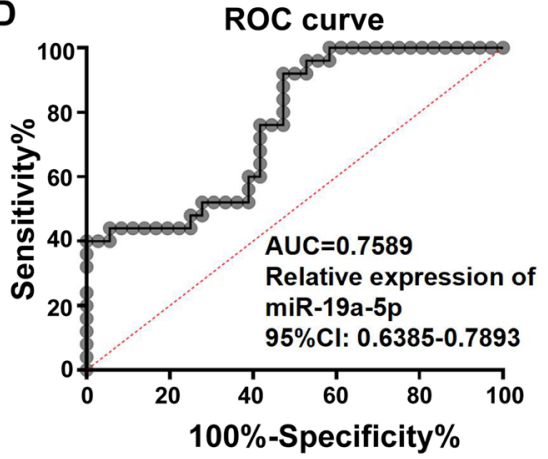

Fig. 3 MiR-19a-5p expression was downregulated in SLE patients and correlated with the disease activities of SLE. a Expression of miR-19a-5p in PBMCs of 36 patients with SLE and 25 healthy controls. b, c Correlations between the expression of miR-19a-5p and the SLEDAl and complement C3 levels were analyzed. $\mathbf{d}$ ROC curve of relative miR-19a-5p expression for distinguishing the 36 patients with SLE from 25 healthy controls. The results are represented as the mean $\pm S D(n=3) .{ }^{* *} P<0.001$

miR-19a-3p. MiR-19a-3p directly targets the $3^{\prime}-\mathrm{UTR}$ of YTHDF2 mRNA. In the following, we attempted to detect whether miR-19a-3p could regulate YTHDF2 expression at the posttranscriptional level. We cloned the $3^{\prime}$-UTR of YTHDF2 mRNA (called psicheck2YTHDF2-wt) and its mutant (called psicheck2YTHDF2-mutant) as shown in Fig. 4d. Luciferase reporter gene assays revealed that miR-19a-3p could weaken luciferase activity by targeting the $3^{\prime}$-UTR of YTHDF2 mRNA. And no decrease in luciferase activity was observed when the target sites were mutated in 293T cells. However, the miR-19a-3p inhibitor resulted in the opposite results (Fig. 4e). Bioinformatics analysis and dual luciferase reporter assays indicated that YTHDF2 was one of the conservative targets of miR19a-3p. Western blotting assay further demonstrated that miR-19a also downregulated the expression of YTHDF2. These results suggest miR-19a might suppress the expression of YTHDF2. Normally, YTHDF2 can identify mRNA m6A sites to regulate mRNA degradation and increase the m6A levels of mRNAs. Taken together, we speculated that circGARS may regulate m6A modification through miR-19a in SLE.
CircGARS directly interacted with m6A-associated proteins to regulate the $\mathrm{A} 20 / \mathrm{NF}-\mathrm{KB}$ signaling pathway

To explore whether circGARS could regulate the A20/ NF-kB signaling pathway, we transfected circGARS overexpression plasmids into PBMCs of SLE patients. The results revealed that the overexpression of circGARS inhibited the expression of A20 and promoted the phosphorylation of $\mathrm{p} 65$ and $\mathrm{IkB \alpha}$. Downregulation of circGARS leaded to decreased phosphorylation of p65 and $\mathrm{IkB} \alpha$ and thereby increased expression of A20 (Fig. 5a). These data demonstrated that circGARS regulates the ubiquitin-editing enzyme A20 to activate the NF- $\mathrm{KB}$ pathway-mediated immune inflammatory response in SLE.

Cytoplasm-localized circRNAs may be involved in translational regulation through competing endogenous RNAs or RNA-binding proteins (RBPs) interactions. Next, we used the SRMAP (http://www.cuilab.cn/sramp) database and detected an m6A motif in circGARS. The secondary structure schematic diagram demonstrated that the motif was located at position 180 (Fig. 5b). Then, we used the database CircInteractome (https://circi nteractome.nia.nih.gov/index.html) and searched RNAbinding proteins matching circGARS (Fig. 5c). The m6A 


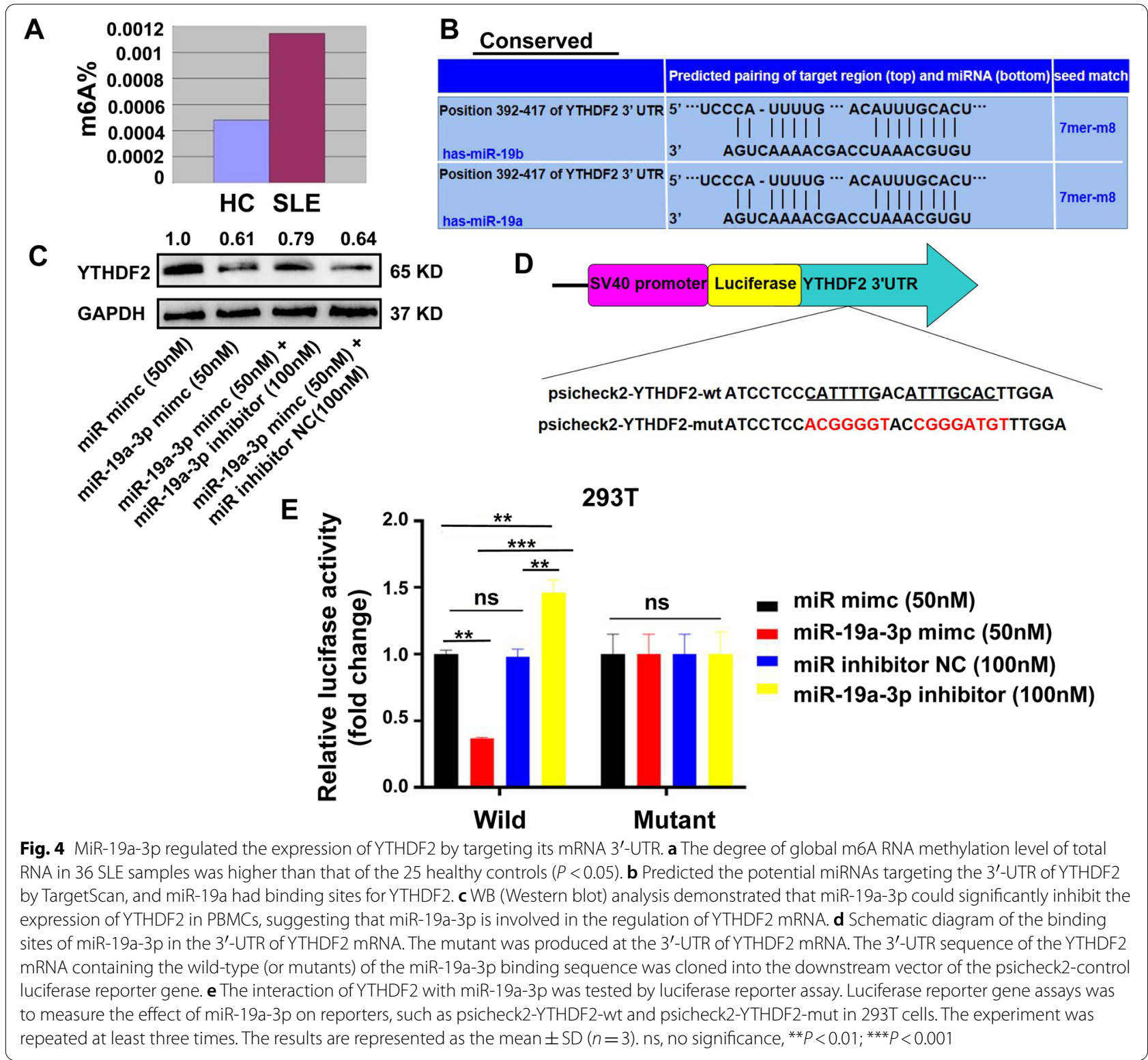

modification protein (HNRNPC) and AGO2 interacted with circGARS, which helps to clarify and understand the role of m6A modification in regulating gene expression of circGARS to a certain extent. Therefore, circGARS directly interacted with m6A-associated proteins to regulate the $\mathrm{A} 20 / \mathrm{NF}-\mathrm{kB}$ signaling pathway.

\section{Discussion}

With the development of high-throughput sequencing technology, more and more attention has been paid to the function of circRNAs. Many reports have shown that the expression of circRNA is closely related to the occurrence of human diseases, especially tumorigenesis [26, 27]. The recent discovery of thousands of circRNAs and their new function in the regulation of gene expression has led us to investigate their role in SLE.

In this study, we screened an upregulated circRNA called circGARS in SLE on the results of RNA-seq and qRT-PCR analysis. We showed that circGARS expression was upregulated in SLE PBMCs and that the expression of circGARS was also correlated with the disease activity of SLE. Therefore, circGARS may be used as a potential biomarker for SLE.

CircRNA is a new type of non-coding RNA with a covalent closed loop structure. In recent years, many 


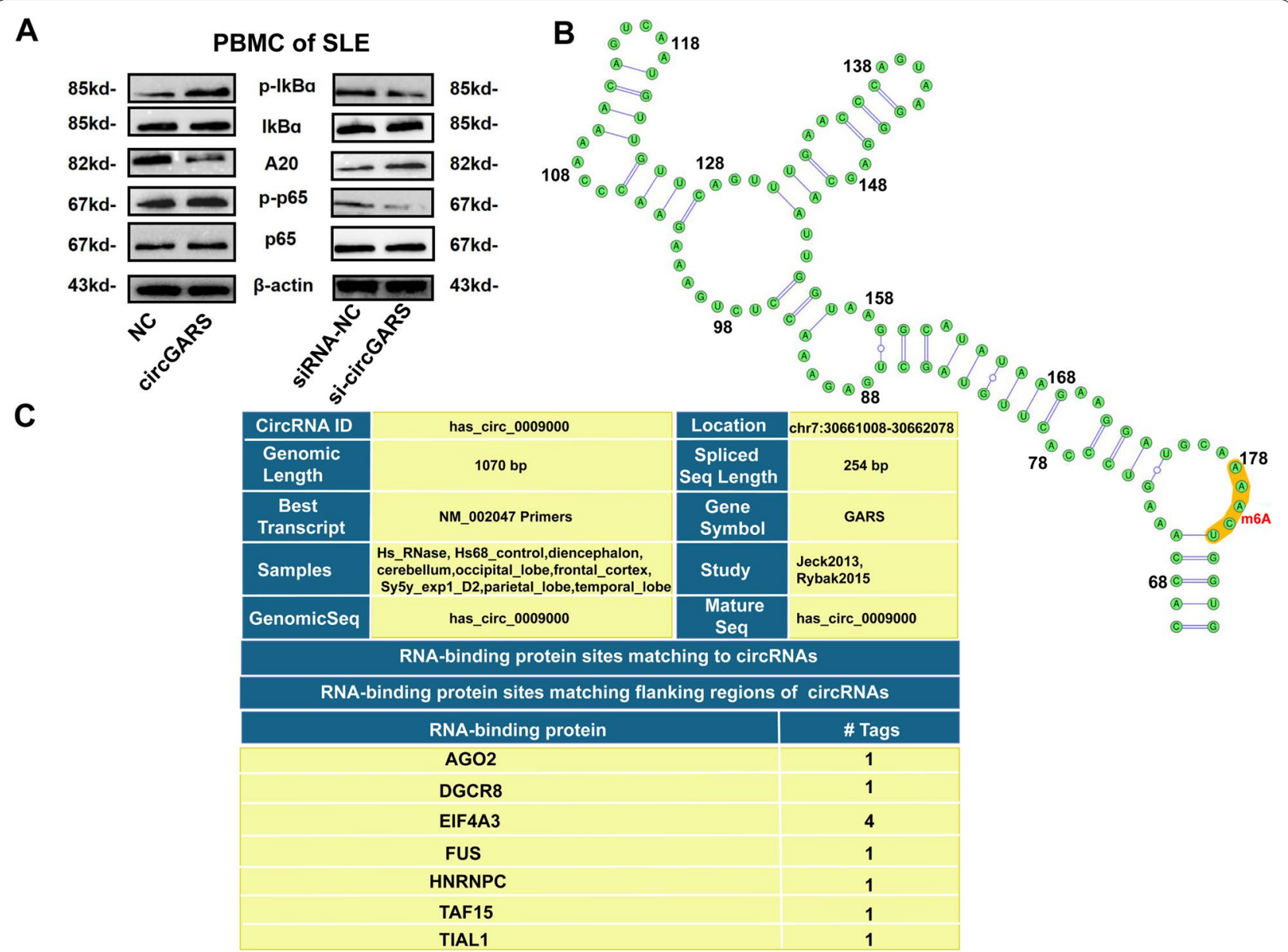

Fig. 5 CircGARS directly interacted with m6A-associated proteins to regulate the A20/NF-kB signaling pathway. a Western blot analysis of A20/ NF-KB signaling-related proteins in PBMCs transfected with circGARS overexpression plasmids, empty vector (NC) si-circGARS, and siRNA-NC in PBMCS of SLE. The results are presented as the mean \pm SD $(n=3)$. $\mathbf{b}$ Image showing the secondary structure of the position of m6A sites located in circGARS. c RNA-binding proteins matching to circGARS

exonic and intronic circRNAs have been discovered in eukaryotes, suggesting that circRNAs are not simply aberrantly spliced by-products but rather have a variety of potential biological functions. The biological functions of circRNAs have been thoroughly studied. CircRNAs can competitively bind miRNAs and regulate the activity of miRNAs on other target genes. For example, circPSMC3 can be used as a new potential biomarker for detecting gastric cancer (GC). CircPSMC3 plays role in the progression of $\mathrm{GC}$ via sponging miRNA-296-5p to modulate the expression of PTEN [28]. Exonic circRNA CDR1as/ciRS-7 contains 63 or 70 binding sites for miR-7 and is a powerful miRNA sponges [29]. More and more evidence has also supported the biological function of circRNAs, such as circRNAs regulate gene expression by interacting with
RNA-binding proteins (RBPs), interfering with microRNA activity and signaling pathways. CircRNAs can also be translated and function as encoded proteins. In addition, circRNAs have potential applications in regulating immune response and cell proliferation as well as in biomedical research. For example, the overexpression of circRNAs containing dsRNA in PBMCs or $\mathrm{T}$ cells in SLE mitigates abnormal PKR activation cascades [30, 31].

Here, bioinformatics analysis showed that circGARS shares the MRE of miR-19a-5p, and luciferase reporter gene assays and RIP experiment verified the direct interaction between circGARS and miR-19a-5p. Further molecular experiments proved that circGARS regulated the ubiquitin-editing enzyme A20 to influence the NF- $\kappa B$ pathway-mediated immune inflammatory 


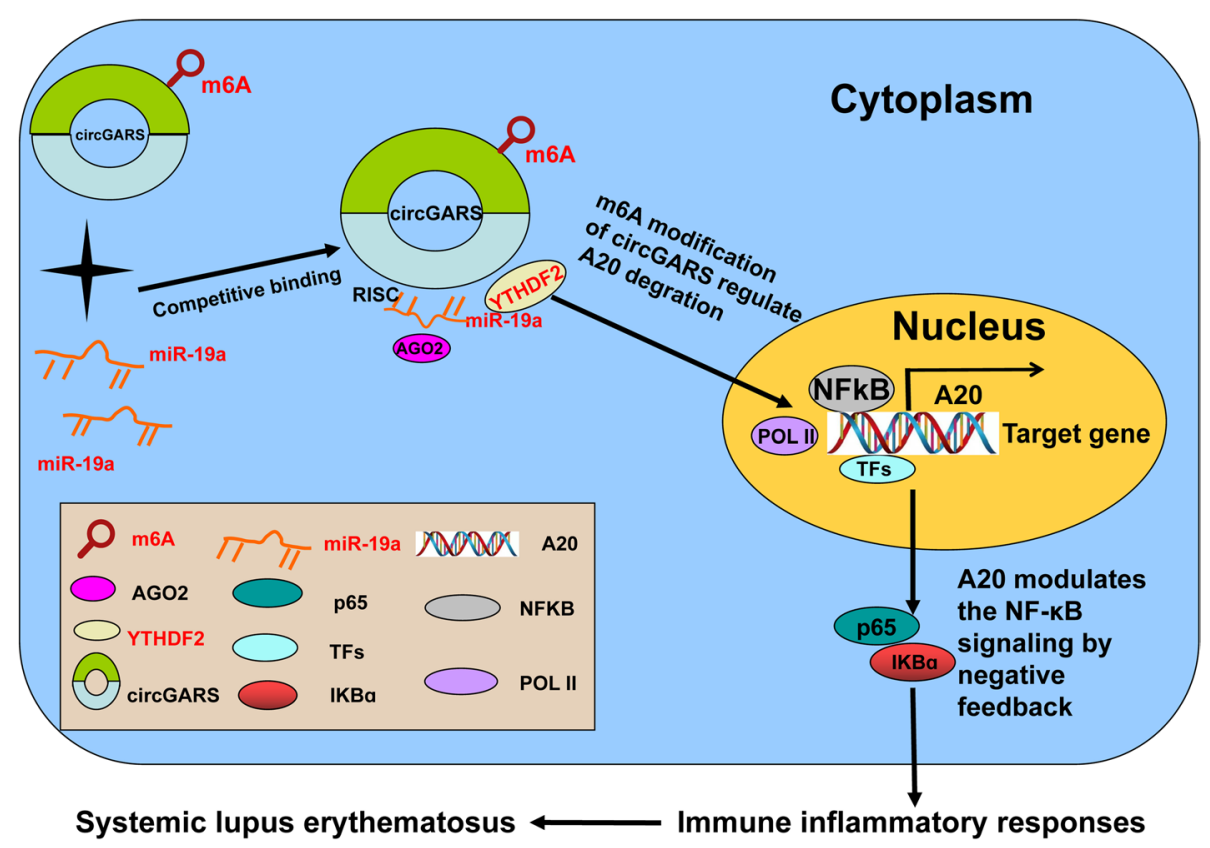

Fig. 6 Schematic diagram of the regulatory mechanism of circGARS in the pathogenesis of SLE. CircGARS directly combined with miR-19a regulate the expression of A20 to influence the immune responses regulated by the NF-KB pathway as a negative feedback mechanism. Moreover, circGARS enhanced the transcriptional level by directly binding to m6A modification proteins. In addition, miR-19a regulated A20 degradation by downregulating YTHDF2

response in SLE. Next, we identified that circGARS interacted with the proteins HNRNPC and the m6A motif in circGARS, suggesting that the function of circGARS occurs through m6A-dependent modification. The function of circRNAs has been extensively studied from different angles. Although m6A is considered to be a rich regulator in mRNAs and ncRNAs, and is involved in many aspects of posttranscriptional mRNA metabolism, researches about the effects of m6A modification on cellular circRNA biology are scarce. N6-methyladenosine (m6A), the most abundant internal modification in mRNA, influences the biological regulation of RNA-protein interactions. YTH domain family 2 (YTHDF2) protein can directly recognize m6A and affect the stabilization of cytoplasmic mRNA [32]. Normally, YTH domain family 1 (YTHDF1) can regulate mRNA translation efficiency by recognizing mRNA $\mathrm{m} 6 \mathrm{~A}$, whereas YTHDF2 is reported to regulate mRNA degradation and cell viability [33-35]. Yang $Z$ et al. reported that microRNA-145 combined with YTHDF2 modulates m6A levels by targeting the $3^{\prime}$-untranslated mRNA region of $\mathrm{m} 6 \mathrm{~A}$, leading to an increase in mRNA methylation [36]. Thus, we further search the mechanism by which circGARS and m6A-dependent modification regulate the expression of genes. Through bioinformatics analysis, we found that YTHDF2 was one of the conservative targets of miR-19a-3p. Herein, dual luciferase reporter assays and western blotting demonstrated that miR-19a shows a distinct regulatory function with YTHDF2 in promoting m6A-modified circGARS, supporting $\mathrm{m} 6 \mathrm{~A}$ as a potential selection signal for mammalian circRNA metabolism. Our results also demonstrate that YTHDF proteins may function as $\mathrm{m} 6 \mathrm{~A}$ modification proteins that promote SLE progression by mediating A20 degradation. It is worth mentioning that the recognition of m6A-modified circRNAs by YTHDF2 do not facilitate their degradation but play a part in segregating m6A-circRNA and inhibiting innate immunity [37]. Similarly, the deletion of METTL3 or YTHDF2 has been reported to stabilize IFNB1 in an m6A-dependent manner following viral infection, leading to subsequent suppression of the virus reproduction in host cell [38]. Previous studies have showed that the complexes of YTHDF2-HRSP12RNase P/MRP and UPF1-G3BP1 may play a key role in regulating the decay of m6A-modified linear mRNAs and degrading mRNAs with a high level of structure in the 3 '-untranslated regions $[39,40]$. The m6A modification is reportedly widespread in circRNAs [41]. However, their precise function remains unknown. 
The function of m6A modification to regulate miRNA processing also makes $\mathrm{m} 6 \mathrm{~A}$ an attractive research subject in SLE. However, whether other miRNAs can direct AGO2 to degrade circRNAs harboring miRNA-binding site(s) remains unknown. Moreover, several miRNAs are involved in the pathogenesis of lupus and show therapeutic potential in SLE [3, 4245]. The homeostasis level of some miRNAs is affected by the knockdown of the expression of m6A demethylase FTO [46], suggesting that the signaling pathways concerning m6A modification with miRNAs should be further explored. How does m6A modification regulate miRNA dysregulated in SLE remains to be determined. Whether and to what extent m6A modification leads to miRNA dysregulation in SLE remains to be elucidated. Research has shown that the binding activities of HNRNPC and m6A-switches will modulate the function of RBP, affecting the gene expression and RNA maturation [32]. The biological regulation of m6A interaction with RNA-protein still needs further clarification. Along with the role of m6A modification in gene expression regulation by modulation of translation, mRNA stability, pre-mRNA splicing, RNA structure, and pri-miRNA processing became increasingly clear. Further study on the role of m6A modification and epitranscriptomics in SLE will promote our understanding of the pathogenesis of SLE.

\section{Conclusions}

Overall, our study discovered a differentially expressed circRNA from patients with SLE, detected the remarkably increased expression of circGARS, and demonstrated that circGARS directly combined with miR-19a-5p. Functionally, circGARS regulated the expression of A20 to influence the inflammatory immune responses regulated by the NF- $\mathrm{kB}$ pathway as a negative feedback mechanism. Moreover, circGARS enhanced the transcriptional level by directly binding to m6A modification proteins. In addition, miR-19a-3p regulated A20 degradation by downregulating YTHDF2. We provide the first evidence that circGARS is a key circRNA related to m6A modification in SLE patients and might be a diagnostic/ prognostic biomarker for SLE (Fig. 6).

\footnotetext{
Abbreviations

SLE: Systemic lupus erythematosus; PBMCs: Peripheral blood mononuclear cells; m6A: N6-methyladenosine; TNFAIP3: Tumor necrosis factor alphainduced protein 3; MiR-19a: MicroRNA-19a; MiRNAs: MicroRNAs; YTHDF1: YTH N6-methyladenosine RNA-binding protein 1; YTHDF2:YTH N6-methyladenosine RNA-binding protein 2; SLEDAl: Systemic Lupus Erythematosus Disease Activity Index; ROC: Receiver operating characteristic; AUC : Area under curve; LncRNAs: Long noncoding RNAs; NF-KB: Nuclear factor-KB; RBPs: RNA-binding proteins; FISH: Fluorescence in situ hybridization; RIP: RNA-binding protein immunoprecipitation; AGO2: Argonaut 2.
}

\section{Supplementary Information}

The online version contains supplementary material available at https://doi. org/10.1186/s13075-022-02732-x.

Additional file 1: Table S1. Detailed information on the SLE patients.

Additional File 2: Table S2. Detailed information on the SLE patients.

Additional File 3: Table S3. Primers for RT-qPCR assays.

Additional File 4: Table S4. Primers for RNA pulldown RT-qPCR assays.

Additional File 5: Figure S1. Volcano plot showing the selected and identified 10 upregulated circRNAs and 10 downregulated circRNAs significantly related with SLE.

Additional File 6: Table S5. Identified and selected 10 upregulated circRNAs and 10 downregulated circRNAs (|fold change $\mid>2, p<0.01)$ between healthy controls and SLE patients by circRNA RNA-seq data.

\section{Acknowledgements}

We thank the patients, nurses, and doctors from the Department of Dermatology and the Traditional Chinese Medicine department of Southwest Hospital for participating in this study.

\section{Authors' contributions}

$X W Z$ and RD mainly performed the research, analyzed the data and wrote the manuscript. YS, LG, JW, ZQS, and YY contributed patient specimens. LLZ and JKG performed the formal analysis, investigation, and statistical analyses. All authors read the manuscript and/or revised it critically. BN provided specialist advice. YY was responsible for the study conception and design. All authors approved the submitted and final version. All authors read and approved the final manuscript.

\section{Funding}

This study was financially supported by the National Natural Science Foundation of China (No. 81673058) and the Natural Science Foundation of Chongqing (cstc2021jcyj-msxm3786).

\section{Availability of data and materials}

The data that support the findings of this study are available from the corresponding author upon reasonable request.

\section{Declarations}

\section{Ethics approval and consent to participate}

This study was approved by the Ethical Committee of the First Affiliated Hospital of Army Medical University and conducted according to Declaration of Helsinki principles. Written informed consent was obtained from all participants. The reference number provided by the ethics committee was KY2020162.

\section{Consent for publication}

Not applicable.

\section{Competing interests}

The authors declared no competing interests.

\section{Author details}

${ }^{1}$ Department of Dermatology, Southwest Hospital, Army Medical University (Third Military Medical University), Chongqing, China. ${ }^{2}$ Chongqing International Institute for Immunology, Chongqing, China. ${ }^{3}$ State Key Laboratory for Conservation and Utilization of Bio-Resources \& Key Laboratory for Microbial Resources of the Ministry of Education, School of Life Science, Yunnan University, Kunming, China. ${ }^{4}$ Department of Traditional Chinese Medicine, Southwest Hospital, Army Medical University (Third Military Medical University), Chongqing, China. ${ }^{5}$ Department of Pathophysiology, College of High Altitude Military Medicine, Army Medical University (Third Military Medical University), Chongqing, China.

Received: 5 August 2021 Accepted: 26 January 2022

Published online: 04 February 2022 


\section{References}

1. Kiriakidou M, Ching CL. Systemic lupus erythematosus. Ann Intern Med. 2020;172(11):ITC81-ITC96.

2. Dörner T, Furie R. Novel paradigms in systemic lupus erythematosus. Lancet. 2019:393(10188):2344-58.

3. Shen N, Liang D, Tang Y, de Vries N. Tak PP. MicroRNAs--novel regulators of systemic lupus erythematosus pathogenesis. Nat Rev Rheumatol. 2012;8(12):701-9.

4. Wu GC, Pan HF, Leng RX, Wang DG, Li XP, Li XM, et al. Emerging role of long noncoding RNAs in autoimmune diseases. Autoimmun Rev. 2015;14(9):798-805.

5. Salzman J. Circular RNA expression: its potential regulation and function. Trends Genet. 2016:32(5):309-16.

6. Memczak S, Jens M, Elefsinioti A, Torti F, Krueger J, Rybak A, et al. Circular RNAs are a large class of animal RNAs with regulatory potency. Nature. 2013;495(7441):333-8.

7. Chen X, Yang T, Wang W, Xi W, Zhang T, Li Q, et al. Circular RNAs in immune responses and immune diseases. Theranostics. 2019;9(2):588-607.

8. Legnini I, Di Timoteo G, Rossi F, Morlando M, Briganti F, Sthandier O, et al. Circ-ZNF609 is a circular rna that can be translated and functions in myogenesis. Mol Cell. 2017;66(1):22-37.e9.

9. Kristensen LS, Andersen MS, Stagsted LVW, Ebbesen KK, Hansen TB, Kjems J. The biogenesis, biology and characterization of circular RNAs. Nat Rev Genet. 2019:20(11):675-91.

10. Luo Q, Zhang L, Li X, Fu B, Guo Y, Huang Z, et al. Identification of circular RNAs hsa_circ_0044235 and hsa_circ_0068367 as novel biomarkers for systemic lupus erythematosus. Int J Mol Med. 2019;44(4):1462-72.

11. Guo G, Wang H, Ye L, Shi X, Yan K, Lin K, et al. Hsa_circ_0000479 as a novel diagnostic biomarker of systemic lupus erythematosus. Front Immunol. 2019;10:2281.

12. Luo Q, Fu B, Zhang L, Guo Y, Huang Z, Li J. Decreased peripheral blood ALKBH5 correlates with markers of autoimmune response in systemic lupus erythematosus. Dis Markers. 2020;2020:8193895.

13. Luo Q, Rao J, Zhang L, Fu B, Guo Y, Huang Z, et al. The study of METTL 14, ALKBH5, and YTHDF2 in peripheral blood mononuclear cells from systemic lupus erythematosus. Mol Genet Genomic Med. 2020;8(9):e1298.

14. Zhao H, Wang L, Luo H, Li QZ, Zuo X. TNFAIP3 downregulation mediated by histone modification contributes to T-cell dysfunction in systemic lupus erythematosus. Rheumatology (Oxford). 2017;56(5):835-43.

15. Wang R, Wang CF, Qin HM, Lu YL, Wei GJ, Huang HT, et al. Association between polymorphisms in the promoter region of miR-17-92 cluster and systemic lupus erythematosus in a Chinese population. J Cell Mol Med. 2018;22(8):4016-20

16. Torri A, Carpi D, Bulgheroni E, Crosti MC, Moro M, Gruarin P, et al. Extracellular MicroRNA signature of human helper T Cell subsets in health and autoimmunity. J Biol Chem. 2017;292(7):2903-15.

17. Chen Y, Wang W, Chen Y, Tang Q, Zhu W, Li D, et al. MicroRNA-19a-3p promotes rheumatoid arthritis fibroblast-like synoviocytes via targeting SOCS3. J Cell Biochem. 2019;120(7):11624-32.

18. Singh PB, Pua HH, Happ HC, Schneider C, von Moltke J, Locksley RM, et al. MicroRNA regulation of type 2 innate lymphoid cell homeostasis and function in allergic inflammation. J Exp Med. 2017;214(12):3627-43.

19. Gantier MP, Stunden HJ, McCoy CE, Behlke MA, Wang D, KaparakisLiaskos $M$, et al. A miR-19 regulon that controls NF-KB signaling. Nucleic Acids Res. 2012:40(16):8048-58

20. Liu M, Wang Z, Yang S, Zhang W, He S, Hu C, et al. TNF-a is a novel target of miR-19a. Int J Oncol. 2011;38(4):1013-22.

21. Adrianto I, Wen F, Templeton A, Wiley G, King JB, Lessard CJ, et al. Association of a functional variant downstream of TNFAIP3 with systemic lupus erythematosus. Nat Genet. 2011;43(3):253-8.

22. Lv X, Liu X, Zhao M, Wu H, Zhang W, Lu Q, et al. RNA methylation in systemic lupus erythematosus. Front Cell Dev Biol. 2021;9:696559.

23. Miao Q, Zhong Z, Jiang Z, Lin Y, Ni B, Yang W, et al. RNA-seq of circular RNAs identified circPTPN22 as a potential new activity indicator in systemic lupus erythematosus. Lupus. 2019;28(4):520-8.

24. Zhou J, Wan J, Gao X, Zhang X, Jaffrey SR, Qian SB. Dynamic m(6)A mRNA methylation directs translational control of heat shock response. Nature. 2015:526(7574):591-4.
25. Guarnerio J, Zhang Y, Cheloni G, Panella R, Mae Katon J, Simpson M, et al. Intragenic antagonistic roles of protein and circRNA in tumorigenesis. Cell Res. 2019;30(2):628-40.

26. Guo X, Zhou Q, Su D, Luo Y, Fu Z, Huang L, et al. Circular RNA circBFAR promotes the progression of pancreatic ductal adenocarcinoma via the miR-34b-5p/MET/Akt axis. Mol Cancer. 2020;19(1):83.

27. Kong Y, Li Y, Luo Y, Zhu J, Zheng H, Gao B, et al. circNFIB1 inhibits lymphangiogenesis and lymphatic metastasis via the miR-486-5p/PIK3R1/ VEGF-C axis in pancreatic cancer. Mol Cancer. 2020;19(1):82.

28. Rong D, Lu C, Zhang B, Fu K, Zhao S, Tang W, et al. CircPSMC3 suppresses the proliferation and metastasis of gastric cancer by acting as a competitive endogenous RNA through sponging miR-296-5p. Mol Cancer. 2019;18(1):25.

29. Xu L, Zhang M, Zheng X, Yi P, Lan C, Xu M. The circular RNA ciRS-7 (Cdrlas) acts as a risk factor of hepatic microvascular invasion in hepatocellular carcinoma. J Cancer Res Clin Oncol. 2017;143(1):17-27.

30. Liu CX, Li X, Nan F, Jiang S, Gao X, Guo SK, et al. Structure and Degradation of Circular RNAs Regulate PKR Activation in Innate Immunity. Cell. 2019;177(4):865-80.e21.

31. Chen LL. The expanding regulatory mechanisms and cellular functions of circular RNAs. Nat Rev Mol Cell Biol. 2020;21(8):475-90.

32. Liu N, Dai Q, Zheng G, He C, Parisien M, Pan T. N(6)-methyladenosinedependent RNA structural switches regulate RNA-protein interactions. Nature. 2015;518(7540):560-4.

33. Wang $X$, Zhao BS, Roundtree IA, Lu Z, Han D, Ma H, et al. N(6)-methyladenosine modulates messenger RNA translation efficiency. Cell. 2015:161(6):1388-99.

34. Du H, Zhao Y, He J, Zhang Y, Xi H, Liu M, et al. YTHDF2 destabilizes m(6) A-containing RNA through direct recruitment of the CCR4-NOT deadenylase complex. Nat Commun. 2016;7:12626.

35. Zhou C, Molinie B, Daneshvar K, Pondick JV, Wang J, Van Wittenberghe $\mathrm{N}$, et al. Genome-wide maps of m6A circRNAs identify widespread and cell-type-specific methylation patterns that are distinct from mRNAs. Cell Rep. 2017;20(9):2262-76.

36. Yang Z, Li J, Feng G, Gao S, Wang Y, Zhang S, et al. MicroRNA-145 modulates N(6)-methyladenosine levels by targeting the 3'-untranslated mRNA region of the N(6)-methyladenosine binding YTH domain family 2 protein. J Biol Chem. 2017;292(9):3614-23.

37. Chen YG, Chen R, Ahmad S, Verma R, Kasturi SP, Amaya L, et al. N6-methyladenosine modification controls circular RNA immunity. Mol Cell. 2019;76(1):96-109.e9.

38. Winkler R, Gillis E, Lasman L, Safra M, Geula S, Soyris C, et al. m(6)A modification controls the innate immune response to infection by targeting type I interferons. Nat Immunol. 2019;20(2):173-82.

39. Guo Y, Wei X, Peng Y. Structure-mediated degradation of CircRNAs. Trends Cell Biol. 2020;30(7):501-3.

40. Fischer JW, Busa VF, Shao Y, Leung AKL. Structure-mediated RNA decay by UPF1 and G3BP1. Mol Cell. 2020;78(1):70-84.e6.

41. Park OH, Ha H, Lee $\mathrm{Y}$, Boo SH, Kwon DH, Song HK, et al. Endoribonucleolytic cleavage of m(6)A-containing RNAs by RNase P/MRP complex. Mol Cell. 2019;74(3):494-507.e8.

42. Liu J, Zhu L, Xie GL, Bao JF, Yu Q. Let-7 miRNAs modulate the activation of NF-KB by targeting TNFAIP3 and are involved in the pathogenesis of lupus nephritis. PloS one. 2015;10(6):e0121256.

43. Liu Y, Dong J, Mu R, Gao Y, Tan X, Li Y, et al. MicroRNA-30a promotes B cell hyperactivity in patients with systemic lupus erythematosus by direct interaction with Lyn. Arthritis Rheum. 2013;65(6):1603-11.

44. Luo S, Liu Y, Liang G, Zhao M, Wu H, Liang Y, et al. The role of microRNA-1246 in the regulation of B cell activation and the pathogenesis of systemic lupus erythematosus. Clin Epigenetics. 2015;7(1):24.

45. Tang Y, Luo X, Cui H, Ni X, Yuan M, Guo Y, et al. MicroRNA-146A contributes to abnormal activation of the type I interferon pathway in human lupus by targeting the key signaling proteins. Arthritis Rheum. 2009:60(4):1065-75.

46. Berulava T, Rahmann S, Rademacher K, Klein-Hitpass L, Horsthemke B. N6-adenosine methylation in MiRNAs. PloS one. 2015;10(2):e0118438.

\section{Publisher's Note}

Springer Nature remains neutral with regard to jurisdictional claims in published maps and institutional affiliations. 